\title{
Comparison of socioeconomic status of parents and Body Mass Index of their in-school adolescents in Cross River state, Nigeria
}

Ekpenyong BN, Essien V, Ndep AO, Onwusaka O and Peter A.

Department of Public Health, University of Calabar

Correspondence: Bernadine Ekpenyong. Email benyita2001@yahoo.com +2348033475138

\begin{abstract}
Background: Overweight and obesity as well as underweight in adolescence are risk factors of chronic diseases that occur in adulthood. The objective of this research was to assess the Body Mass Index of inschool adolescents and its relationship to socioeconomic status of their parents.

Methods: This is a cross-sectional study of 454 adolescents aged 10-19 years selected from five secondary schools using multistage sampling technique. A semi-structured interviewer questionnaire was used to collect relevant information from the adolescents and their anthropometric measurement to determine their Body Mass Index. Their Body Mass Index was categorized into obese, overweight, normal and underweight using the International Obesity Task Force reference. Analysis was done using IBM Statistical Package for Social Sciences, version 20.1 .

Results: The prevalence of obesity, overweight, and underweight among the adolescents was $7.7 \%, 1.1 \%$ and $32.8 \%$ respectively. None of the students in the public schools was obese while $2.1 \%$ of the adolescents were obese in the private schools. Adolescents in public schools had a higher percentage of underweight (65.4\%) compared to those in private schools $(34.5 \%)$ and this was found statistically significant $(p<0.001)$. There was an overall higher prevalence of overweight and obesity among the females than found among the males. This was also the case for adolescents from higher socio-economic parents. These differences were found to be statistically significant $(p<0.001)$.

Conclusion: The Body Mass Index status of the in-school adolescents studied was found to increase with increased socioeconomic status of their parents. This underscores the need for sensitization of in-school adolescents to see the need of knowing their nutritional status and ways to avoid the consequences of abnormal Body Mass Index.
\end{abstract}

Key words: Body Mass Index, Socioeconomic status, In-school adolescents

Introduction

High Body Mass Index (BMI) in adolescents across populations is on the increase and a cause of concern as this will lead to unhealthy population overtime. ${ }^{1,2}$ The increase in the percentage of children and adolescents who are obese is not just an indication of an increase in the morbidity and mortality of a population but also a health indicator for the measurement of the health status of the entire population. ${ }^{1,2,3}$ The nutritional status 
of adolescents contributes significantly to the health status of the community in which they reside. One of the major global health problems faced by developing countries today is under nutrition. ${ }^{4}$

The World Health Organization (WHO) describes obesity as a global epidemic ${ }^{5}$ and as such the need to assess the prevalence and to find the factors responsible so as to implement timely interventions. The consequences of underweight on the other hand are as grievous as that of overweight. Furthermore, in all adolescents, short stature resulting from chronic undernutrition is associated with reduced body mass and deficiencies in muscular strength and working capacity. ${ }^{4}$ Adolescent obesity which is becoming prevalent, is most of the time due to untreated childhood obesity. This increase is worldwide and in developing countries especially in the urban populations it is an emergent major health issue. ${ }^{6}$ The report of a systematic review on the global prevalence of overweight and obesity has confirmed that the prevalence of BMI of $25 \mathrm{~kg} / \mathrm{m}^{2}$ or greater among adolescents is on the increase, with the prevalence being more among boys in the developed countries and more among girls in the developing countries. $^{2}$

Most studies on the relationship between Socioeconomic Status (SES) and BMI of adolescents were carried out in developed countries and results show that an inverse relationship exist between the two variables. $^{7,8}$ That of developing countries are few with inconsistent results. Majority however reported a positive relationship between SES and BMI status of adolescents, which means that as the SES increases, the BMI increases. ${ }^{9,10}$ There is paucity of published work done on the relationship between SES of Nigerian parents and prevalence of overweight and obesity among their adolescent children. In a study conducted among adolescents in Ondo State, Nigeria, higher prevalence of underweight $(16 \%)$ was more than overweight among the adolescents. The prevalence of overweight was more among adolescents in the urban than those in the rural areas of Ondo state in Nigeria. ${ }^{11}$ The prevalence rate of obesity, overweight and underweight in a study carried out in southern Nigeria was $11.4 \%, 2.8 \%$ and $13.8 \%$ respectively. ${ }^{12}$ In another study the prevalence of obesity among adolescents in Cross River State Nigeria was $4 \%{ }^{13}$ These studies carried in Nigeria did not look at the association between BMI status of adolescents and socioeconomic status of their parents.

Adolescents are the future of any population and their health status is a reflection of the expected health status of the population in the nearest future. This study was conducted to assess the relationship between the SES of parents and the BMI of their adolescents in public and private schools in Calabar, Cross River State, Nigeria. This will help to provide database in the study area which can be used to formulate preventive strategies to mitigate the consequences of abnormal BMI.

\section{Methods}

The study was a descriptive crosssectional study done in Calabar, Cross River State, Nigeria between July and August 2016. The sample size 454 was determined by the application of minimum sample size formula, $n=Z^{2} p / d^{2}$ for a prevalence of normal BMI from previous study of $6.67 \%{ }^{14}$ Multistage sampling technique was used to select the adolescents aged 10 -19 years old. The choice of selecting three private schools and two public schools was based on the fact that there are more private schools 
than public schools in the study area. Selection of participants was by proportionate sampling based on the population of each school selected through simple random sampling by balloting. The list of public and private schools in Calabar municipality was used as the sampling frame for the selection. In each of the schools selected, one class each was selected through simple random sampling technique by balloting from JS1, JS2, SS1 and SS2. Students in JS3 and SS3 were excluded because they were taking external examinations at the time of the study. In each selected class, students 1019 years old were selected through simple random sampling technique by balloting on the list of students in each class.

A pre-tested semi-structured interviewer administered questionnaire was used to collect the data before their anthropometric measurements were done. The anthropometric measurement tools were inspected and calibrated for use. The BMI of participants was determined using the Quetelet formula of weight $(\mathrm{kg}) /$ height $\left(\mathrm{m}^{2}\right)$. The BMI was compared to the percentile level for the age of the participants and was compared to the standard BMI classification. Underweight was defined as BMI less than or equal to $18.5 \mathrm{~kg} / \mathrm{m}^{2}$ Overweight as BMI $25-$ $29.9 \mathrm{~kg} / \mathrm{m}^{2}$ while from $30 \mathrm{~kg} / \mathrm{m}^{2}$ and above as obese. ${ }^{13}$ Data collected from the field were analyzed using the Statistical Package for Social Sciences (SPSS) software version 20.1. Socioeconomic status of parents was computed by assigning scores to parents' education level and occupation, which were graded using the $25^{\text {th }}, 50^{\text {th }}$ and $75^{\text {th }}$ percentile as low, medium and high Scores respectively.
Chi-square test statistics was used to analyze the association between variables and p-value less than 0.05 was considered statistically significant. Ethical approval was obtained from the Department of Public Health Ethics Committee, University of Calabar, before research commenced. Also, written informed consent was obtained from their parents, while verbal assent was obtained from the students after clear explanation of the research to the student before data collection.

\section{Results}

Out of the 454 students who participated in the study $48 \%$ were males, $51.2 \%$ were from Private Schools and the mean age of all the participants was $14.0 \pm 2.4$ years (Table 1). The study showed the BMI of the adolescents as $35.7 \%$ underweight, $7.7 \%$ overweight while $1.1 \%$ were obese. (Figure 1). The prevalence of overweight and obesity among the females were $11.8 \%$ and $2.1 \%$ respectively and $3.1 \%$ and $0 \%$ among the males respectively. The males were more underweight $(46.3 \%)$ than the females $(25.8 \%)$. The difference was statistically significant $(\mathrm{P}<0.001)$. (Table 2) Comparing the BMI of the inschool adolescents in the private school with those in public schools showed that $49.1 \%$ and $47.5 \%$ in public schools were underweight with none obese while $24.2 \%$ and $2.1 \%$ were underweight and obese respectively in the private schools. This was statistically significant $(p<0.001)$. The relationship between the socioeconomic status of the parents and the BMI of their adolescents showed that adolescents from high socioeconomic status were more obese $(p<0.001)$. However, there was no statistically significant difference between the high BMI and age groups $(\mathrm{p}=0.09)$ (Table 2). 
Table 1. Demographic characteristics of respondents $(n=454)$

\begin{tabular}{llr}
\hline Variables & Group & Freq. $(\%)$ \\
\hline Age (years) & $10-12$ & $79(17.4)$ \\
& $13-15$ & $265(58.4)$ \\
& $16-18$ & $102(22.4)$ \\
& $19-21$ & $8(1.8)$ \\
Sex & Male & $218(48.0)$ \\
\multirow{3}{*}{ School } & Female & $236(52.0)$ \\
& Private & $229(51.2)$ \\
Class & Public & $225(48.8)$ \\
& JS 1 & $100(22.0)$ \\
& JS 2 & $118(26.0)$ \\
& SS 1 & $123(27.1)$ \\
& SS 2 & $113(24.9)$ \\
\hline
\end{tabular}

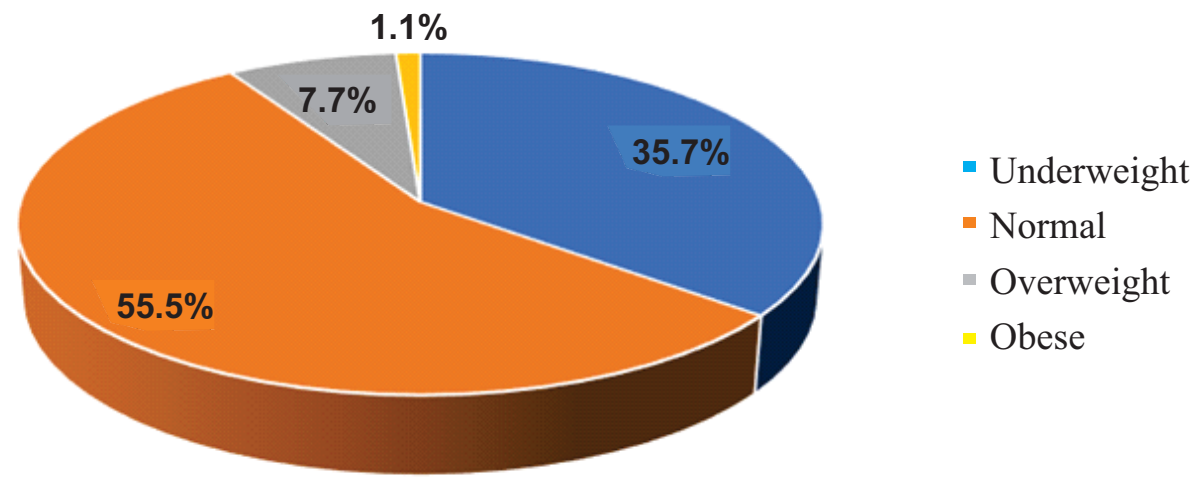

FIG 1. Classification of BMI Status of adolescents in Cross River State Nigeria

Table 2. Body Mass Index by age, sex, school type and socioeconomic status $(n=454)$

\begin{tabular}{|c|c|c|c|c|c|c|c|}
\hline Variables & Group & $\begin{array}{l}\text { Underweight } \\
\text { Freq.(\%) }\end{array}$ & $\begin{array}{l}\text { Normal } \\
\text { Freq.(\%) }\end{array}$ & $\begin{array}{l}\text { Overweight } \\
\text { Freq.(\%) }\end{array}$ & $\begin{array}{l}\text { Obese } \\
\text { Freq. } \%\end{array}$ & $\begin{array}{l}\text { Total } \\
\text { Freq. (\%) }\end{array}$ & $\begin{array}{l}\text { P- } \\
\text { value } \\
\end{array}$ \\
\hline \multirow[t]{4}{*}{ Age (years) } & $10-12$ & $27(36.9)$ & $48(60.7)$ & $4(5.0)$ & $0(0.0)$ & $79(17.4)$ & 0.090 \\
\hline & $13-15$ & $140(52.8)$ & $154(58.1)$ & $21(7.9)$ & $3(1.1)$ & $265(58.4)$ & \\
\hline & $16-18$ & $45(44.1)$ & $48(47.0)$ & $10(9.8)$ & $1(0.9)$ & $102(22.4)$ & \\
\hline & $19-21$ & $5(62.5)$ & $2(25)$ & $0(0.0)$ & $1(12.5)$ & $8(1.8)$ & \\
\hline \multirow[t]{2}{*}{ Sex } & Male & $101(46.3)$ & $110(50.5)$ & $7(3.1)$ & $0(0.0)$ & $218(48.0)$ & $<0.001$ \\
\hline & Female & $61(25.8)$ & $142(60.1)$ & $28(11.8)$ & $5(2.1)$ & $236(52.0)$ & \\
\hline \multirow[t]{2}{*}{ School } & Private & $56(24.2)$ & $148(64)$ & $22(9.5)$ & $5(2.1)$ & $231(50.8)$ & $<0.001$ \\
\hline & Public & $106(47.5)$ & 104 (46.6) & $13(5.8)$ & $0(0.0)$ & $223(49.1)$ & \\
\hline \multirow[t]{3}{*}{ SES of Parents } & Low & $92(82.8)$ & $19(17.1)$ & $9(8.1)$ & $0(0.0)$ & $111(24.5)$ & $<0.001$ \\
\hline & Medium & $56(22.6)$ & $162(65.5)$ & $26(10.5)$ & $1(0.4)$ & $247(54.4)$ & \\
\hline & High & $14(14.5)$ & $71(73.1)$ & $9(9.3)$ & $4(4.1)$ & $96(21.1)$ & \\
\hline
\end{tabular}

SES - Socioeconomic status 


\section{Discussion}

Abnormal BMI is a growing public health problem which requires early detection and management for a healthy population although this study shows a higher prevalence of underweight when compared with that of overweight and obesity among the in-school adolescents. This finding agrees with similar studies in Nigeria that looked at both overweight and underweight among adolescents.,11, 12 However, a previous study in Calabar, Cross River state had reported a higher prevalence of overweight and obesity. ${ }^{12}$ The higher prevalence of underweight reported in this study, when compared to other studies in Nigeria, could be linked to prevailing poor economic situation in Nigeria. ${ }^{11,14}$

The relationship between sex and high BMI in this study is similar to studies carried out within and outside the country. In the developing countries, a prevalence of overweight and obesity of $12.9 \%$ among boys and $13.4 \%$ among girls; and a prevalence of $23.8 \%$ and $22.6 \%$ for boys and girls respectively in the developed countries has been reported in a systematic review. ${ }^{2}$ This showed that females in the developing countries had higher BMI than their male counterpart, while the reverse was the case for developed countries. ${ }^{2,5,16}$ In a study carried out in Kano, it was shown that the difference between the prevalence of overweight in females and males were not significant, but in this study and several others, the difference between overweight and sex was statistically significant. 2,14,15, ${ }_{17,18,19}$ However, it is clear that sex is associated with BMI status of adolescents, as such parents with female children should pay more attention to their nutritional needs and encourage physical exercise.

Socioeconomic status of parents was associated with BMI status of their adolescents. Those from high socioeconomic status parents were more obese than those from low to medium socioeconomic status while adolescents from low socio-economic status parents were more underweight.

The higher prevalence of underweight seen in the public schools could be that the adolescents were mostly from parents of low economic status as public schools are more affordable to them as compared to the private schools. This result is in line with findings from similar studies carried out in other developing countries. ${ }^{9,10}$ The reverse is the case in studies from the western countries that found that SES was inversely associated with BMI of children and adolescents. ${ }^{7,8}$ The difference could be due to variations in the lifestyle of people in the developing and developed nations. Obese and overweight children are likely to stay obese into adulthood and are more likely to develop non-communicable diseases like diabetes and cardiovascular diseases at a younger age. The prevalence of underweight is still a condition of public health importance and was more prevalent among adolescents from low SES. This finding is similar to the study done in Osun State Nigeria which showed higher prevalence of underweight among children in public than private schools, and higher underweight in adolescents in rural secondary schools than those in urban secondary schools. ${ }^{14}$ This may be that parents of high SES are more likely to send their children to private schools. It is therefore important for parents to be educated on how to plan healthy diets for their children irrespective of their SES.

\section{Conclusion}

Abnormal BMI is becoming prevalent among in-school adolescents in Calabar municipality. This study also shows a high prevalence of underweight among in- 
school adolescents in the population as well as a low but growing prevalence of overweight and obesity. The prevalence of high BMI was higher in private schools, adolescents from high SES and females. The prevalence of underweight was more among adolescents in public schools with low SES. The need to start sensitization on consequences of abnormal BMI cannot be overemphasized as the health status of adolescents serves as a health indicator for the entire population, reasons being that they are the future adults of any population. Health education on nutrition and how to avoid abnormal BMI should be emphasized in schools while healthy school feeding programs should be extended to public secondary schools where such does not exist.

\section{References}

1. Sahoo K, Sahoo B, Choudhury AK, Sofi NY, Kumar R, Bhadoria AS. Childhood obesity: causes and consequences. J Family Med Prim Care. 2015;4(2):187-192. doi:10.4103/2249-4863.154628

2. Ng M, Fleming $\mathrm{T}$, Robinson $\mathrm{M}$, Thomson B, Graetz N, Margono C, Mullany EC. Global, Regional and National Prevalence of overweight and Obesity in Children and Adults during 1980-2013: A Systematic Analysis for the Global Burden of disease study in 2013. The Lancet. 2014;384(9945):766-81.

3. Melaku YA, Zelo GA, Gill TK, Shi, RJ. Prevalence and factors associated with stunting and thinness among adolescent students in Northern Ethiopia: a comparison to World Health Organization standards. Arch Public Health. 2015; 73 (44): 2-11.

4. Esimai OA, Ojofeitimi EO. Nutrition and Health Status of Adolescents in a Private Secondary School in Port Harcourt. Health Sciences Journal.
2015; 9 (5): 1-5.

5. Prasanna KBT, Bengalorkar GM, Deepthi R, Muninarayan C, Ravishankar S. Prevalence of overweight and obesity among adolescent school going children (12$15 y e a r s)$ in urban area, south india. International Journal of Current Research and Review. 2012; 4(20): 99-105.

6. Mandal A, Mandal GC. Prevalence of overweight and obesity among urban adolescent English medium school girls of Kolkata India. Italian Journal of Public Health. 2012; 9(3): 4.

7. Morgenstern M, Sargent JD, Hanewinkel R. Relation between socioeconomic status and body mass index: evidence of an indirect path via television use. Archives of Pediatrics and Adolescent Medicine. 2009; 163(8): 731-8.

8. Hanson MD, Chen E. Socioeconomic Status, Race, and Body Mass Index: The Mediating Role of Physical Activity and Sedentary Behaviors during Adolescence, Journal of Pediatric Psychology. 2007; 32(3): 250-259. https://doi.org/10.1093/jpepsy/js1024

9. Kim J, Sharma SV, Park SK. Association between socioeconomic status and obesity in adults: evidence from the 2001 to 2009 Korea national health and nutrition examination survey. Journal of Preventive Medicine and Public Health. 2014; 47(2): 94-103.

10. Ahmed A, Zulaily N, Shahril MR, Syed Abdullah EFH, Ahmed A.

Association between socioeconomic status and obesity among 12-year-old Malaysian adolescents. PLoS ONE. 2018; 13(7): e0200577. https://doi.org/10.1371/journal. pone. 0200577

11. Mustapha RA, Sanusi RA. 
Overweight and Obesity among Inschool Adolescents in Ondo State, Southwest Nigeria. African Journal of Biomedical Research. 2013;16(3):205-210.

12. Ene-Obong H, Ibeanu V, Onuoha N, Ejekwu A. Prevalence of overweight, obesity, and thinness among urban school-aged children and adolescents in southern Nigeria.

Food and Nutrition Bulletin, 2012; 33(4) 242-250.

13. Ansa VO, Odigwe CO, Anah MU. Profile of body mass index and obesity in Nigerian children and adolescents. Nigerian Journal of Medicine. 2001; 10(2): 78 -80.

14. Olumakaiye MO. Prevalence of Underweight: A Matter of Concern among Adolescents in Osun State, Nigeria. Pakistan Journal of Nutrition .2008; 7 (3): 503-508.

15. Ahmad MM, Ahmed H, Airede K. Body Mass Index among School Adolescents in Sokoto, North-Eastern
Nigeria. Sahel Medicine Journal. 2013; 16(1): 5-9.

16. WHO. Obesity and overweight key facts 2018. Accessed Feb 2019https://www.who.int/news/factsh eets/details. 2019Kanter R

17. , Caballero B. Global gender disparities in obesity: a review. Adv Nutr. 2012; 3(4):491-8. doi: 10.3945/an.112.002063.

18. Yusuf SM, Mijinyawa MS, Musa BM, Gezawa ID, Uloko AE. Overweight and Obesity among Adolescents in Kano, Nigeria. J Metabolic Synd. 2013; 2 (1): 126. doi:10.4172/2167-0943.1000126

19. Musa DI, Toriola AL, Monyeki MA, Lawal B. Prevalence of childhood and adolescent overweight and obesity in Benue State, Nigeria. Tropical Medicine and International Health.2013;17(11):1369-1375. 
